\title{
Late Diagnosis of Wilson Disease, Initially Presenting as Cerebellar Atrophy Mimicking Spinocerebellar Ataxia, by Multigene Panel Testing
}

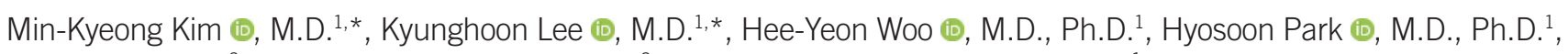

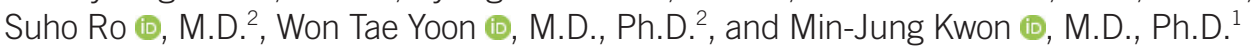

Departments of ${ }^{1}$ Laboratory Medicine and ${ }^{2}$ Neurology, Kangbuk Samsung Hospital, Sungkyunkwan University School of Medicine, Seoul, Korea

\section{Dear Editor,}

Wilson disease (WD; OMIM \#277900) is an autosomal recessive disorder associated with ATP7B variants. WD can manifest as multi-organ disturbances due to excessive copper accumulation and symptoms usually present between the ages of five and 35 years. Early manifestations of WD are easily overlooked and misdiagnosed as hepatitis, cirrhosis, splenomegaly, or encephalitis, leading to delayed treatment. If patients are diagnosed early and managed properly, permanent organ damage can be effectively prevented [1-3]. Asymptomatic or atypical WD diagnosis is still difficult, and few reports have diagnosed WD cases using next generation sequencing (NGS)-based gene panel test $[4,5]$. We report a case of atypical WD mimicking spinocerebellar ataxia (SCA), diagnosed using an NGS panel test. Written informed consent for genetic test was obtained from the patient, his parents, and sister. This study was approved for waiver of informed consent by the Institutional Review Board of Kangbuk Samsung
Hospital, Seoul, Korea (KBSMC 2018-10-018).

A 30-year-old male presented with a six-month history of bradykinesia and progressive gait instability. The neurological examination at admission on May 2018 indicated that he had limb ataxia, cerebellar type dysarthria, gait disturbance, and gait instability. Serum levels of aspartate aminotransferase (AST, 0.55 $\mu \mathrm{kat} / \mathrm{L}$ ), alanine aminotransferase (ALT, $0.48 \mu \mathrm{kat} / \mathrm{L}$ ), total bilirubin (t-bil, $12.5 \mu \mathrm{mol} / \mathrm{L}$ ), and gamma-glutamyltransferase (GGT, $1 \mu \mathrm{kat} / \mathrm{L})$ were all normal. Brain magnetic resonance imaging (MRI) showed mild cerebellar atrophy without typical MRI findings, such as hyperintense lesions involving the putamen, pons, midbrain, and the thalamus, on the T2-weighted image (Fig. 1) [6]. Based on the cerebellar atrophy, MRI finding, and a history of bradykinesia and progressive gait instability, SCA was suspected.

Genetic testing of the SCA type 1, 2, 3, 6, 7, and 8 gene profile and DRPLA, as well as fluoro-propyl-carbomethoxy-iodophe-
Received: January 29, 2020

Revision received: March 18, 2020

Accepted: May 21, 2020

Corresponding author: Min-Jung Kwon, M.D., Ph.D.

Department of Laboratory Medicine, Kangbuk Samsung Hospital,

Sungkyunkwan University School of Medicine, 29 Saemunan-ro, Jongnogu, Seoul 03181, Korea

Tel: +82-2-2001-5211, Fax: +82-2-757-0711, E-mail: mjkkmd@gmail.com

Co-corresponding author: Won Tae Yoon, M.D., Ph.D.

Department of Neurology, Kangbuk Samsung Hospital, Sungkyunkwan

University School of Medicine, 29 Saemunan-ro, Jongno-gu, Seoul 03181,

Korea

Tel: +82-2-2001-2467, Fax: +82-2-2001-2988, E-mail: wtyoon@gmail.com

\section{(c) (1) (8)}

(C) Korean Society for Laboratory Medicine

This is an Open Access article distributed under the terms of the Creative Commons Attribution Non-Commercial License (https://creativecommons.org/licenses/by-nc/4.0) which permits unrestricted non-commercial use, distribution, and reproduction in any medium, provided the original work is properly cited.

*These authors contributed equally to this study. 

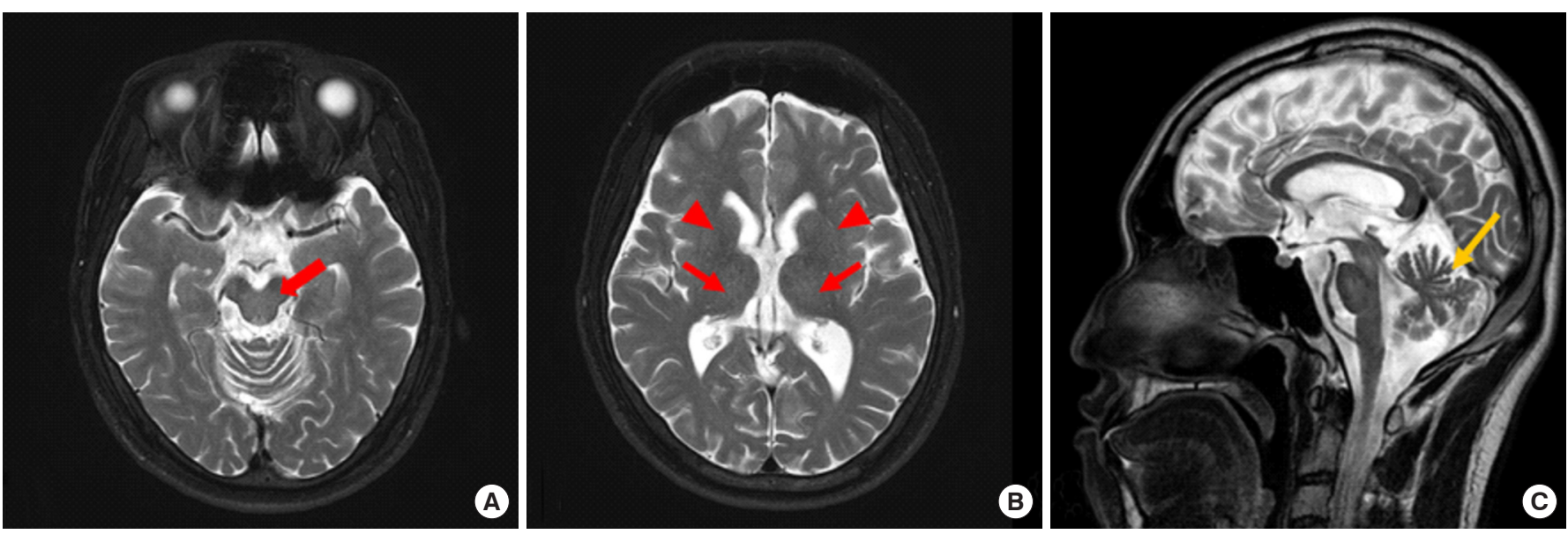

Fig. 1. T2-weighted magnetic resonance images of the patient. (A) The axial image does not show high signal intensity in the midbrain (red arrow). (B) The axial image does not show high signal intensity in the thalamus (red arrows) or putamen (red arrowheads).(C) The sagittal image shows cerebellar atrophy (yellow arrow).

nyl-tropane positron emission tomography were conducted; all results were normal. In addition, an ataxia panel testing was conducted to distinguish other rare genetic diseases and hereditary ataxia.

Genomic DNA was extracted from the patient's peripheral blood. The targeted gene panel included 293 genes spanning a 1,159,873 base pair (bp) region based on the hg19 human reference genome (https://www.ncbi.nlm.nih.gov/assembly/GCF_000001405.13/). Exomes were captured using a customized Target Enrichment System Kit (Celemics, Seoul, Korea). NGS was performed using the MiSeqDX platform (Illumina Inc., San Diego, CA, USA) with $150 \mathrm{bp}$ paired-end sequencing, using the following pipeline: Burrows-Wheeler Aligner MEM algorithm 0.7.12 (https://arxiv. org/abs/1303.3997), Genome Analysis Toolkit 3.5 [7], Variant Effect Predictor (https://asia.ensembl.org/info/docs/tools/vep/index.html), dbNSFP (http://varianttools.sourceforge.net/Annotation/dbNSFP), SAMtools 0.1.19 (https://sourceforge.net/projects/ samtools/files/samtools/0.1.19/), FreeBayes 0.9.2.1 (https:// github.com/ekg/freebayes), and Scalpel 0.5.3 (http://scalpel. sourceforge.net/) and Picard tools 1.96 (https://broadinstitute. github.io/picard/) for data analysis. The NGS results identified a homozygous missense variant, NM_000053.3: c.3104G > T (p.Gly1035Val), in exon 14 of ATP7B, and this variant was classified as likely pathogenic.

Although we did not suspect WD during the clinical work-up owing to late onset age, normal AST, ALT, and MRI findings, WD was genetically diagnosed based on the ataxia panel testing performed by NGS. After two months, the results of the additional tests we performed were as follows: the presence of Kayser-Flei- scher (KF) rings under slit-lamp examination, decreased serum ceruloplasmin (45 mg/L [reference range, 200-600 mg/L]) and

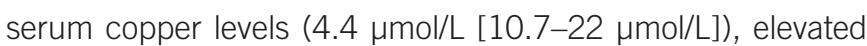

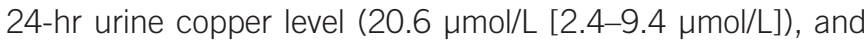

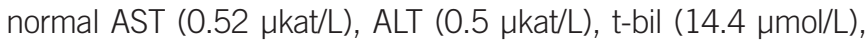
and $\mathrm{r}$-GGT levels (1.06 $\mu \mathrm{kat} / \mathrm{L})$. Additionally, we re-evaluated the WD score following genetic test confirmation; the total WD score was 10 points (two points for the presence of KF rings, two for higher urine copper level, two for decreased serum ceruloplasmin level, zero for the absence of typical WD brain MRI result and hemolytic anemia, and four for the presence of the variant on both chromosomes) [8]. Finally, the patient was genetically and clinically diagnosed as having WD. Sanger sequencing of ATP7B among the patient's family revealed that his father, mother, and sister harbored a heterozygous C.3104G > T variant and were asymptomatic carriers (Fig. 2).

The patient received oral penicillamine therapy at a dosage of 250 mg/day from September 2018, and his neurological symptom improved after treatment. The patient has been followed-up for one year under outpatient care with penicillamine therapy. One year later, the patient's 24-hour urine copper level is within the stable range, and his health condition is good without any side effects.

WD is known to have a higher genetic prevalence than clinical prevalence, with a carrier frequency of approximately 1:90 based on the Hardy-Weinberg equilibrium; approximately $40 \%$ of WD patients have an obvious neurological presentation, ranging from movement disorders or tremors to rigid dystonia-like gait disturbance [9]. In Korea, the frequency of WD and its genetic carri- 


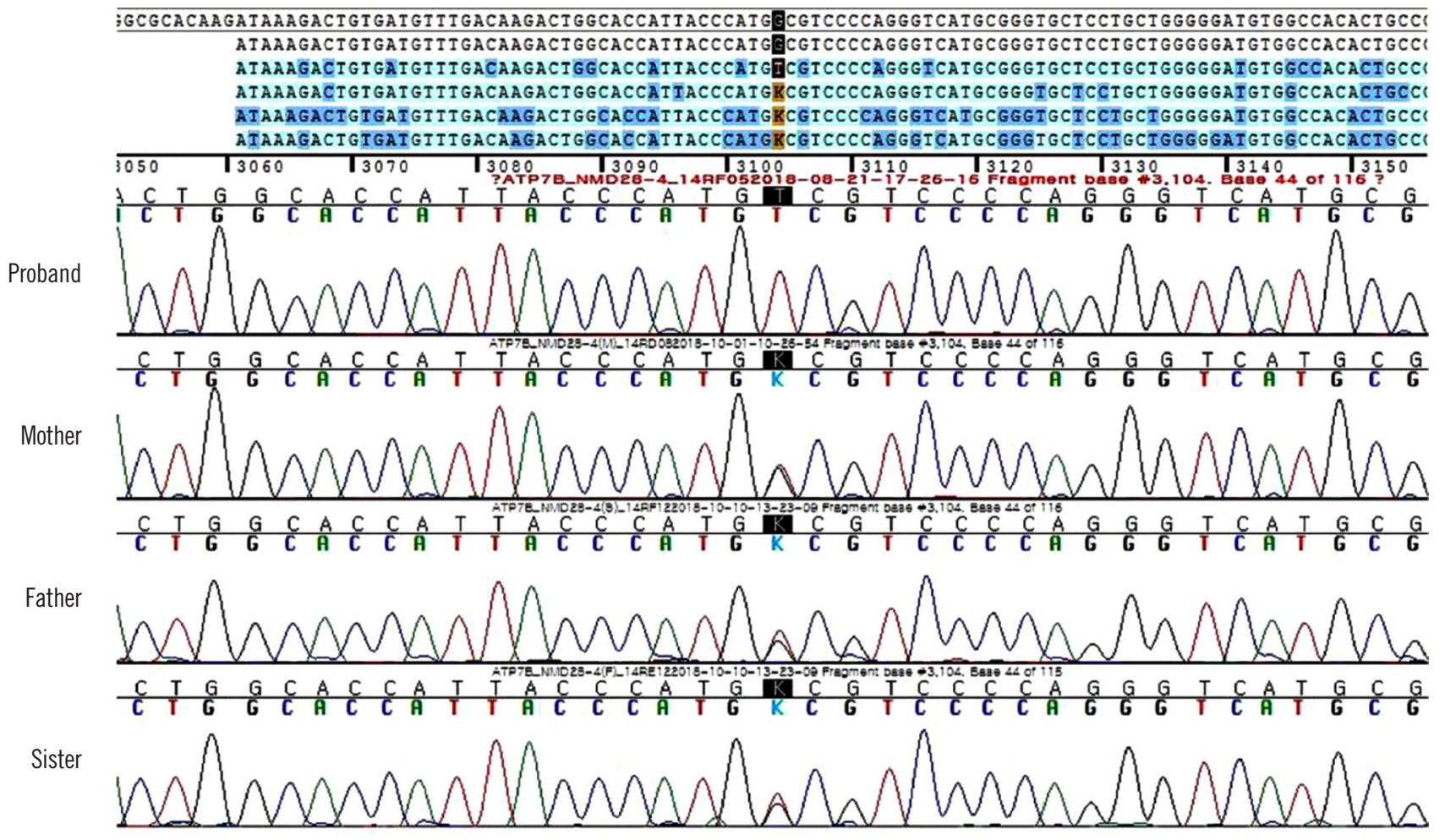

Fig. 2. Sanger sequencing of the proband's family revealed that his parents and sister are heterozygous for the variant detected in the proband.

ers has been reported as 1:53, higher than in other countries [10]. In addition, the disease may be underdiagnosed, suggesting the need for WD screening in high-risk populations [10]. An NGS panel including ATP7B might be helpful for the differential diagnosis of neurological diseases.

NGS was a useful and reliable diagnostic method in our atypical WD patient. We suggest that NGS could be helpful in the case of atypical WD phenotypes. As WD can present with cerebellar ataxia and without Parkinsonism, brain MRI abnormalities, or elevated liver enzyme levels, it should be considered in all young patients with atypical hepatic or neurological symptoms. Moreover, it is important to include WD-associated genes when constructing a neurogenetic panel.

\section{ACKNOWLEDGEMENTS}

None declared.

\section{AUTHOR CONTRIBUTIONS}

MJK designed this study and edited the manuscript; MKK and $\mathrm{KL}$ acquired and analyzed the data and substantially contrib-

uted to the writing of the manuscript; SR and WTY provided clinical information regarding the patient; and HP, HYW, and WTY reviewed the patient's clinical information and reviewed the manuscript critically. All authors approved the final manuscript.

\section{CONFLICTS OF INTEREST}

No potential conflicts of interest relevant to this article were reported.

\section{RESEARCH FUNDING}

This work was supported by Medical Research Funds from Kangbuk Samsung Hospital.

\section{ORCID}

Min-Kyeong Kim Kyunghoon Lee Hee-Yeon Woo Hyosoon Park https://orcid.org/0000-0002-2843-7285 https://orcid.org/0000-0002-5014-3179 https://orcid.org/0000-0002-1154-3137 https://orcid.org/0000-0002-5400-4205 
Kim MK, et al.

Late diagnosis of Wilson disease by NGS

Suho Ro

https://orcid.org/0000-0003-3378-561X

Won Tae Yoon

https://orcid.org/0000-0002-7026-3899

Min-Jung Kwon

https://orcid.org/0000-0002-2372-0700

\section{REFERENCES}

1. Tanzi RE, Petrukhin K, Chernov I, Pellequer JL, Wasco W, Ross B, et al. The Wilson disease gene is a copper transporting ATPase with homology to the Menkes disease gene. Nat Genet 1993;5:344-50.

2. Rosencrantz R, Schilsky M. Wilson disease: pathogenesis and clinical considerations in diagnosis and treatment. Semin Liver Dis 2011;31: 245-59.

3. Prashanth LK, Taly AB, Sinha S, Arunodaya GR, Swamy HS. Wilson's disease: diagnostic errors and clinical implications. J Neurol Neurosurg Psychiatry 2004;75:907-9.

4. Poujois A and Woimant F. Challenges in the diagnosis of Wilson disease. Ann Transl Med 2019;7(S2):S67.

5. Ye S, Dai T, Leng B, Tang L, Jin L, Cao L. Genotype and clinical course in 2 Chinese Han siblings with Wilson disease presenting with isolated disabling premature osteoarthritis: a case report. Medicine (Baltimore) 2017;96:e8641.

6. Kim TJ, Kim IO, Kim WS, Cheon JE, Moon SG, Kwon JW, et al. MR imaging of the brain in Wilson disease of childhood: findings before and after treatment with clinical correlation. AJNR Am J Neuroradiol 2006; 27:1373-8.

7. McKenna A, Hanna M, Banks E, Sivachenko A, Cibulskis K, Kernytsky A, et al. The Genome Analysis Toolkit: a MapReduce framework for analyzing next-generation DNA sequencing data. Genome Res 2010;20: 1297-303.

8. Ferenci P, Caca K, Loudianos G, Mieli-Vergani G, Tanner S, Sternlieb I, et al. Diagnosis and phenotypic classification of Wilson disease. Liver Int 2003;23:139-42.

9. Ala A, Walker AP, Ashkan K, Dooley JS, Schilsky ML. Wilson's disease. Lancet 2007;369:397-408.

10. Jang JH, Lee T, Bang S, Kim YE, Cho EH. Carrier frequency of Wilson's disease in the Korean population: a DNA-based approach. J Hum Genet 2017;62:815-8. 